Paper

\title{
Development of nonlinear analysis tools on FPGA
}

\author{
Shogo Shimada ${ }^{1 a)}$ and Hiroyuki Kitajima ${ }^{1}$ \\ ${ }^{1}$ Department of Electronics and Information Engineering, Kagawa University \\ 2217-20 Hayashi, Takamatsu, Kagawa 761-0396, Japan \\ a) s15g468@stu.kagawa-u.ac.jp
}

Received June 22, 2016; Revised October 13, 2016; Published April 1, 2017

\begin{abstract}
Many mathematical models in various scientific fields are represented by complicated differential equations, and such models cannot be solved analytically. Therefore, numerical analysis using computers is essential. However, when a system is more complicated, the analysis time is longer. In this study, we propose a fast numerical analysis method using a field programmable gate array (FPGA). The FPGA is a reconfigurable integrated circuit, and it is good at parallel processing. We developed the calculators of bifurcation diagrams and basins of attraction on an FPGA board. As a result, we constructed calculators that were at most 2.8 times faster and that consumed $95.2 \%$ less power than the conventional method using a CPU.
\end{abstract}

Key Words: bifurcation, dynamical system, FPGA, numerical analysis

\section{Introduction}

Mathematical models are important for capturing the essence of complicated natural phenomena. We consider one of the most important mathematical models to be the Hodgkin-Huxley neuron model [1]. Since the publication of this model, many conductance-based neuron models have been proposed. Among them, we are interested in cardiac cell models because the suppression of arrhythmia through studies of mathematical models can reduce the risk of sudden cardiac arrest. A recent model of the ventricular cell including complicated calcium dynamics consists of 45 ordinary differential equations (ODEs) [2]. Usually, such mathematical models are described by nonlinear functions. We cannot solve them analytically. Thus, numerical analysis using computers is effective. In numerical analysis, high computational costs (calculation time and electric power) are required for higher accuracy which requires an ODE solver with less error and a more detailed mathematical model. Thus, we must construct fast calculators with low electric power consumption.

In nonlinear dynamical systems, we observe complicated phenomena such as quasi-periodic states, chaotic states, and co-existence of attractors. The generation of these phenomena is related to bifurcations. A bifurcation is defined by the qualitative change of solutions due to a perturbation of parameter values. It is very important to understand the bifurcation structure in parameter space because dynamical diseases such as arrhythmia, Cheyne-Stokes respiration, and chronic granulocytic leukemia are caused by bifurcations [3-5]. However, studying parameter space in a complicated dynamical system (high-dimensional dynamical system) takes a lot of time because the transient time to a steady state becomes longer, and we should investigate bifurcations in many combinations of pa- 
rameters contained in such a system. If the attractors of the system coexist, so called "biexcitability" in cardiac models [6-8], then the basin of attraction gives us useful information on the initial state dependency of the attractors. A plane of initial states on a grid is considered, and we check which attractor is obtained from each initial point. We give each attractor a distinct color. Usually, the basin boundary gives a stable manifold of a saddle type periodic solution on the boundary. Thus, using the basin, we can visualize the global structure of stable manifolds. Moreover, its fractalization is related to homoclinic points $[9,10]$, and its qualitative change is related to synchronization [11]. The calculation of basin of attraction for high-dimensional systems also requires much computational costs.

Here, we use an FPGA device to overcome this difficulty. The FPGA is a reconfigurable integrated circuit, and it is good at parallel processing [12]. Using this advantage, simulators of neuron models [13-15], chaos generators $[16,17]$, and neural networks $[18,19]$ have been constructed on FPGA devices. We previously proposed a method of bifurcation analysis for simple maps on an FPGA [20]. In this paper, we design nonlinear analysis tools for calculating bifurcation diagrams and basins of attraction using an FPGA. In a numerical experiment, we show that our method is faster than the conventional numerical calculation using a CPU.

\section{Preliminaries}

\subsection{Fixed-point operation}

In this research, we use 32-bits $(1,7$, and 24 bits are used for the sign, integer, and fractional parts, respectively) fixed-point number representation for operation on an FPGA. (Floating point calculation is used for software. $)^{1}$ The FPGA used here has multipliers with 18 bits. We use two kinds of multipliers: our own multiplier using the Booth algorithm and a multiplier equipped on the FPGA. The former and latter are denoted by "Booth" and "multiplier" in Sec. 3, respectively. The multiplier on the FPGA is faster than the Booth method; however, accuracy becomes worse. Thus, we compare these methods on the FPGA to check the relationship between calculation speed and accuracy. The CORDIC algorithm [21] is used for the calculation of mathematical functions.

\subsection{Model equations}

Duffing's equations, which describe the dynamics of an oscillatory circuit with a nonlinear inductor [22], are given by

$$
\begin{aligned}
& \frac{d x}{d t}=y, \\
& \frac{d y}{d t}=-k y-c_{3} x^{3}+B_{0}+B \cos \omega t .
\end{aligned}
$$

We fix the parameter values as $k=0.1, c_{3}=1.0, \omega=1$, and $B_{0}=-0.075$.

The Bonhöffer-van der Pol (BVP) model [23] with an external force is given by

$$
\begin{aligned}
& \frac{d x}{d t}=c\left(y+x-\frac{1}{3} x^{3}+h \sin \omega t\right), \\
& \frac{d y}{d t}=-\frac{1}{c}(x+b y-a) .
\end{aligned}
$$

We fix the parameter values as $a=0.0, b=0.4$, and $c=1.5$. This model was proposed as a simplification of the Hodgkin-Huxley neuron model, and the bifurcation structures of the single model [24, $25]$ and the coupled models [26-28] were widely studied.

We define the Poincaré map for Eqs. (1) and (2) as

$$
T: R^{2} \rightarrow R^{2} ; \xi \mapsto T(\xi, \lambda)=\psi(2 \pi / \omega, \xi, \lambda),
$$

where $\xi=[x, y]^{\mathrm{T}}, \lambda$ is a parameter vector, and $\psi\left(t, \xi_{0}, \lambda\right)$ is assumed to be a solution of Eq. (1) or (2) with an initial condition $\xi_{0}=\left[x_{0}, y_{0}\right]^{\mathrm{T}}$ at $t=t_{0}$. Then, the $l$-periodic point $\xi^{*}$ of Eq. (1) or (2) is defined as

\footnotetext{
${ }^{1}$ Floating-point calculation is faster than fixed-point calculation for a CPU in our test.
} 
Table I. Results for Duffing's equations.

\begin{tabular}{c|c|c|c|c|c}
\hline & $\begin{array}{c}\text { degree of } \\
\text { parallelism }\end{array}$ & $\begin{array}{c}\text { runtime } \\
(\text { hh:mm:ss })\end{array}$ & $\begin{array}{c}\text { occupied LE } \\
(\max 114480)\end{array}$ & $\begin{array}{c}\text { multiplier } \\
(\max 532)\end{array}$ & $\begin{array}{c}\text { electric } \\
\text { power[Wh] }\end{array}$ \\
\hline \hline \multirow{2}{*}{ software } & 1 & $01: 49: 07$ & - & - & 54.6 \\
\cline { 2 - 6 } & 2 & $00: 54: 33$ & - & - & 33.6 \\
\hline \multirow{2}{*}{$\begin{array}{c}\text { FPGA } \\
\text { Booth) }\end{array}$} & 1 & $15: 28: 27$ & 5387 & 0 & 66.5 \\
\cline { 2 - 6 } & 2 & $7: 44: 22$ & 7919 & 0 & 33.3 \\
\hline \multirow{2}{*}{$\begin{array}{c}\text { FPGA } \\
\text { (multiplier) }\end{array}$} & 1 & $00: 19: 27$ & 113388 & 0 & 1.62 \\
\cline { 2 - 6 } & 2 & $16: 00: 59$ & 3909 & 20 & 68.9 \\
\cline { 2 - 6 } & 32 & $08: 00: 55$ & 5406 & 36 & 34.5 \\
\hline
\end{tabular}

$$
\xi^{*}-T^{l}\left(\xi^{*}, \lambda\right)=0 .
$$

We calculate bifurcation diagrams and basins of attraction for the BVP and Duffing's equations, respectively. Both figures are obtained through the following procedure:

1. a plane of parameters (bifurcation diagram) or initial states (basin of attraction) on a grid is considered, then initial states in bifurcation diagrams and parameter values in basins of attraction are fixed,

2. we check which attractor is obtained from each point on the grid after 1000 iterations of the Poincaré map,

3. we give each attractor a distinct color.

\section{Results}

We used a computer (CPU: i5-560m with clock frequency of $2.66 \mathrm{GHz}$ ) for software calculation (compiler: Borland bcc55) and an FPGA board (DE2-115) for hardware calculation. An FPGA [Cyclone IV E: clock frequency of $50 \mathrm{MHz}$ with 114,480 logic elements (LE) and 532 multipliers] was equipped on the board. This board had a VGA port, so results (diagrams) are shown on a display through this port.

\subsection{Basin of attraction}

Figures 1, 2, and 3 show the basins of attraction for Duffing's equations using the software, FPGA (Booth), and FPGA (multiplier), respectively. A fixed point and two-periodic points coexisted at $B=0.15$. From the initial states colored by blue, we obtained the fixed point as an attractor. Two-periodic points were obtained from the initial states colored by red and green. Increasing the value of $B$ to 0.185 , four-periodic points appeared as a result of the period-doubling bifurcation of the two-periodic points. The boundaries of four colors were fractalized because of the appearance of homoclinic points [29] as shown in, for example, Fig. 1(b). Comparing between the results of the software and the FPGAs, the shape of each colored region was almost the same; for example, the degree of agreement of Figs. 1(a) and 2(a) is 99.8\%. We consider that the reason of the small difference comes from the fixed-point calculation for the FPGA. Thus, the FPGAs can calculate the basin of attraction. Table I shows a comparison of runtime and electric power for the three methods. When the degree of parallelism was one, the software was faster than the FPGAs. The FPGA (Booth) and FPGA (multiplier) could have 48 and 32 parallel processings at most using an optimization tool on Quartus II (optimize for area in Resource Optimization Adviser). Thus, runtimes were measured to be about 19 and 30 minutes, which is 2.8 and 1.8 times faster than using software, respectively. The power consumption of each circuit was also measured by using a watt-hour meter (SHW3A: System Works Co.), and we determined that the power consumption of the FPGA (Booth) was 20 times lower than that of the CPU. The FPGA has the advantage of faster calculation and of using less electric power compared with the CPU. 


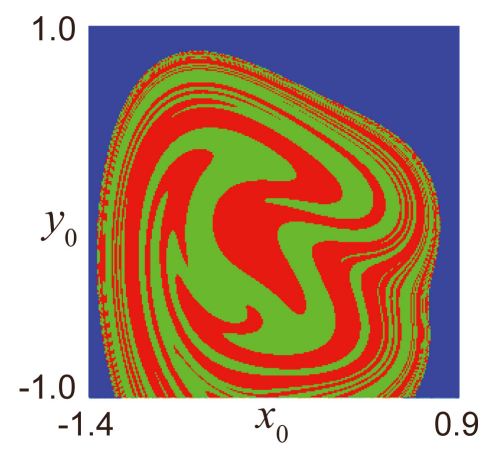

(a) $B=0.15$.

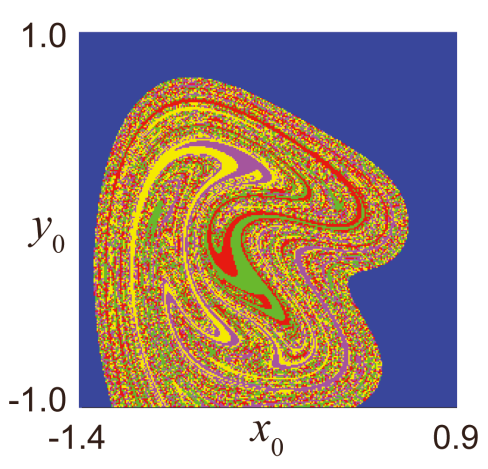

(b) $B=0.185$.

Fig. 1. Basin of attraction for Duffing's equations obtained by using software.

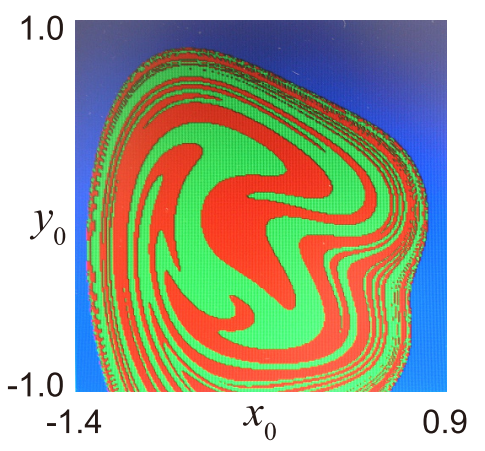

(a) $B=0.15$.

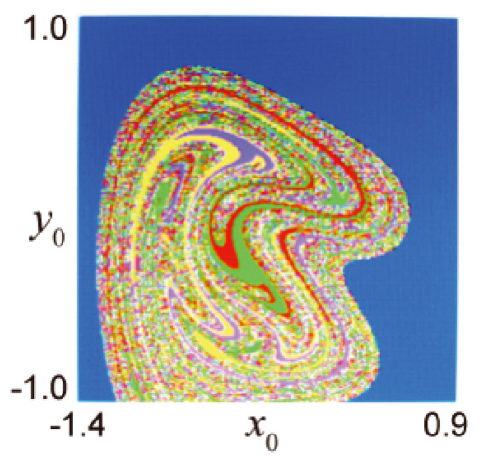

(b) $B=0.185$

Fig. 2. Basin of attraction for Duffing's equations obtained by using FPGA (Booth).

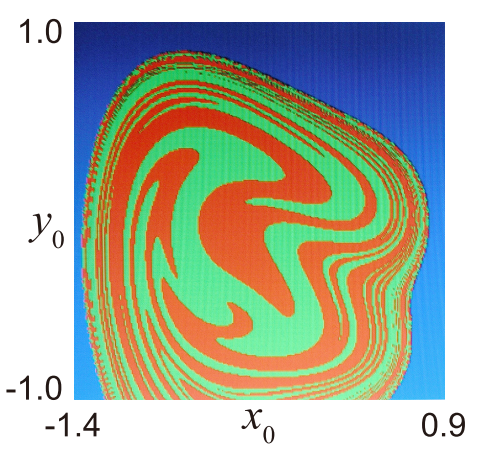

(a) $B=0.15$

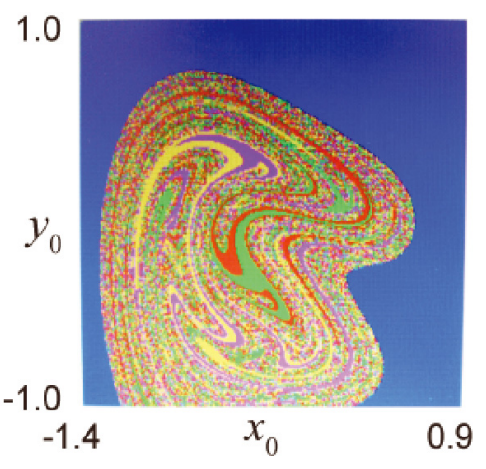

(b) $B=0.185$.

Fig. 3. Basin of attraction for Duffing's equations obtained by using FPGA (multiplier). 
Table II. Color assignment.

\begin{tabular}{c|c||c|c}
\hline period & color & period & color \\
\hline \hline 1 & blue & 7 & white \\
\hline 2 & red & 8 & slate blue \\
\hline 3 & magenta & 9 & pink \\
\hline 4 & green & 10 & purple \\
\hline 5 & cyan & $\begin{array}{c}\text { non-period or } \\
\text { divergence }\end{array}$ & black \\
\hline 6 & yellow & \\
\hline
\end{tabular}

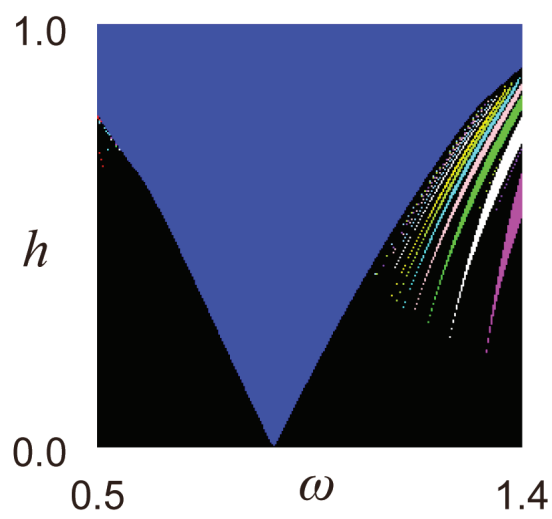

(a) Software

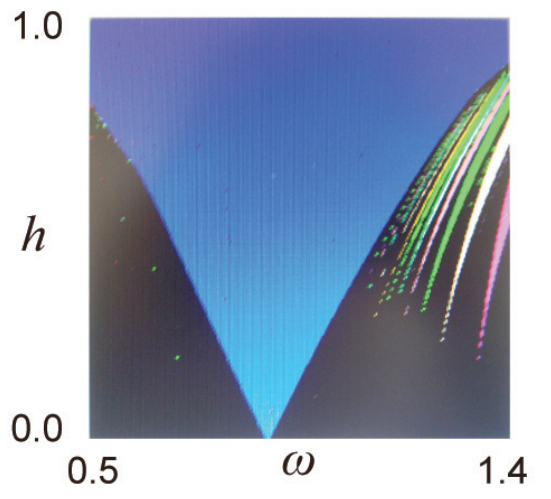

(b) FPGA (Booth)

Fig. 4. Bifurcation diagram for BVP model.

Table III. Results for BVP model.

\begin{tabular}{c|c|c|c|c}
\hline & $\begin{array}{c}\text { degree of } \\
\text { parallelism }\end{array}$ & $\begin{array}{c}\text { runtime } \\
\text { (hh:mm:ss })\end{array}$ & $\begin{array}{c}\text { occupied LE } \\
(\max 114480)\end{array}$ & $\begin{array}{c}\text { electric } \\
\text { power[Wh] }\end{array}$ \\
\hline \hline \multirow{2}{*}{ software } & 1 & $02: 17: 14$ & - & 68.6 \\
\cline { 2 - 5 } & 2 & $01: 08: 57$ & - & 42.3 \\
\hline \multirow{2}{*}{ FPGA } & 1 & $22: 55: 50$ & 6148 & 98.6 \\
\cline { 2 - 5 } (Booth) & 2 & $11: 27: 50$ & 9282 & 49.3 \\
\cline { 2 - 5 } & 40 & $00: 34: 20$ & 114093 & 2.86 \\
\hline
\end{tabular}

\subsection{Bifurcation diagram}

We show the results of calculating bifurcation diagrams for the BVP model. The meaning of colors is shown in Table II [30]. From the result of the previous subsection, we can see that there is no merit in using a multiplier on the FPGA for both speed and power consumption. Figures 4(a) and 4(b) represent the result of using the software and the FPGA (Booth), respectively. The degree of agreement of these figures is $99.9 \%$. The BVP model without the external force has an oscillatory solution at the parameter values we decided. The parameter region of the entrainment of the frequency for the oscillatory solution is colored blue. The other colors except black indicate the regions where stable sub-harmonic oscillations existed. The results of runtime are shown in Table III. When the degree of parallelism was changed from 1 to 2, the occupation of LE in the FPGA raised 2.7\%. Thus, theoretically, 32 degrees of parallelism is possible. Using an optimization tool on Quartus II (optimize for area in Resource Optimization Adviser), we can achieve 40 parallelisms with $99.67 \%$ occupation. This is 2.0 times faster than using the software. We succeed in reducing power consumption by $93.2 \%$.

\section{Conclusion}

We developed nonlinear analysis tools on an FPGA. Calculators of bifurcation diagrams and basins of attraction were constructed on the FPGA. We determined the accuracy of our calculators and showed that our method is faster than the conventional method using a CPU. Also, the calculator on 
the FPGA has the advantage of using less electric power. One application of our method is to search the dominant parameters of generating arrhythmia in mathematical cardiac models. For example, there are 50 parameters in the Shannon model [2]. Studying the parameter plane for such a system, 1225 parameter planes must be checked in total. Our method can capture the properties of solutions in nonlinear systems at low cost. However, using a hardware description language such as Verilog HDL or VHDL to write a program is not easy for someone who has not studied hardware description languages. Therefore, we intend to make a user-friendly tool that will enable users to easily use hardware description languages by simply rewriting the differential equations. Moreover, we are now investigating the use of OpenCL [31] to combine different devices such as CPUs, GPUs, DSPs, and FPGAs to achieve faster calculation. Until now we obtained that FPGA (Terasic DE5-Net) is 15 times faster than CPU (one core of Intel Xeon E5-2697) and GPU (NVIDIA Quadro 410) is 6 times faster than the FPGA using Open CL. We can make a very high performance computer for analyzing nonlinear systems by using the FPGA, GPU, multi-core of the CPU.

\section{Acknowledgments}

This work was supported by JSPS KAKENHI (15K00405). We thank Altera Co. for providing the FPGA boards (DE2-115 and DE5-Net) through the University Program.

\section{References}

[1] A.L. Hodgkin and A.F. Huxley, "A quantitative description of membrane current and its application to conduction and excitation in nerve," The Journal of Physiology, vol. 117, no. 4, pp. 500-544, 1952.

[2] T.R. Shannon, F. Wang, J. Puglisi, C. Weber, and D.M. Bers, "A mathematical treatment of integrated Ca dynamics within the ventricular myocyte," Biophysical Journal, vol. 87, pp. 3351$3371,2004$.

[3] M. Mackey and L. Glass, "Oscillation and chaos in physiological control system," Science, vol. 197, pp. 287-289, 1997.

[4] T. Krogh-Madsen and D.J. Christini, "Nonlinear dynamics in cardiology," Annual Rev. Biomed. Eng., vol. 14, pp. 179-203, 2012.

[5] L. Glass, "Dynamical disease: Challenges for nonlinear dynamics and medicine," Chaos, vol. 25, no. 9, 097603, 2015.

[6] M.G. Chang, D. Sato, E. de Lange, J.H. Lee, H.S. Karagueuzian, A. Garfinkel, and Z. Qu, "Bistable wave propagation and early afterdepolarization-mediated cardiac arrhythmias," Heart rhythm, vol. 9, no. 1, pp. 115-122, 2012.

[7] M.G. Chang, E. de Lange, G. Calmettes, A. Garfinkel, Z. Qu, and J.N. Weiss, "Pro-and antiarrhythmic effects of ATP-sensitive potassium current activation on reentry during early afterdepolarization-mediated arrhythmias," Heart Rhythm, vol. 10, no. 4, pp. 575-582, 2013.

[8] N. Vandersickel, I.V. Kazbanov, A. Nuitermans, L.D. Weise, R. Pandit, and A.V. Panfilov, "A study of early afterdepolarizations in a model for human ventricular tissue," PloS one, vol. 9, no. 1, e84595, 2014.

[9] C. Mira, D. Fournier-Prunaret, L. Gardini, H. Kawakami, and J.C. Cathala, "Basin bifurcations of two-dimensional noninvertible maps: fractalization of basins," Int. J. Bifurcation and Chaos, vol. 4, no. 2, pp. 343-381, 1994.

[10] H. Kitajima, H. Kawakami, and C. Mira, "A method to calculate basin bifurcation sets for a twodimensional noninvertible map," Int. J. Bifurcation and Chaos, vol. 10, no. 8, pp. 2001-2014, 2000.

[11] P.J. Menck, J. Heitzig, N. Marwan, and J. Kurths, "How basin stability complements the linearstability paradigm," Nature Physics, vol. 9, no. 2, pp. 89-92, 2013.

[12] G. Constantinides, A.B. Kinsman, and N. Nicolici, "Numerical data representations for FPGAbased scientific computing," IEEE Design and Test of Computers, vol. 28, no. 4, pp. 8-17, 2011. 
[13] E.L. Graas, E.A. Brown, and R.H. Lee, "An FPGA-based approach to high-speed simulation of conductance-based neuron models," Neuroinformatics, vol. 2, no. 4, pp. 417-435, 2004.

[14] R.K. Weinstein and R.H. Lee, "Architectures for high-performance FPGA implementations of neural models," Journal of Neural Engineering, vol. 3, no. 1, pp. 21-34, 2005.

[15] K. Tateno, "A FPGA-based two-dimensional neuron model and its bifurcation," IEICE Tech. Repo., vol. NC2010-49, pp. 41-45, 2010 (in Japanese).

[16] M.A. Zidan, A.G. Radwan, and K.N. Salama, "The effect of numerical techniques on differential equation based chaotic generators," Proc. IEEE/ICM'2011, pp. 1-4, December 2011.

[17] W. Guang-Yi, B. Xu-Lei, and W. Zhong-Lin, "Design and FPGA Implementation of a new hyperchaotic system," Chinese physics B, vol. 17, no. 10, 3596, 2008.

[18] B. Glackin, T. M. McGinnity, L.P. Maguire, Q.X. Wu, and A. Belatreche, "A novel approach for the implementation of large scale spiking neural networks on FPGA hardware," in Computational Intelligence and Bioinspired Systems, eds. J. Cabestany, A. Prieto, and F. Sandoval, pp. 552-563, Springer, Heidelberg, 2005.

[19] A.R. Omondi and J. C. Rajapakse (eds.), FPGA implementations of neural networks, Springer, Dordrecht, 2006.

[20] J. Tada, T. Yamaguchi, and H. Kitajima, "Development of FPGA simulator for bifurcation analysis," Proc. NCSP'13, pp. 45-48, March 2013.

[21] J.E. Volder, "The CORDIC trigonometric computing technique," IRE Trans. Electron. Comput., vol. EC-8, pp. 330-334, 1959.

[22] C. Hayashi, Nonlinear Oscillations in Physical Systems, Princeton University Press, 1964.

[23] R. Fitzhugh, "Impulses and physiological states in theoretical models of nerve membrane," Biophysical Journal, vol. 1, pp. 445-466, 1961.

[24] S. Sato and S. Doi, "Response characteristics of the BVP neuron model to periodic pulse inputs," Mathematical Biosciences, vol. 112, no. 2, pp. 243-259, 1992.

[25] S. Doi and S. Sato, "The global bifurcation structure of the BVP neuronal model driven by periodic pulse trains," Mathematical Biosciences, vol. 125, no. 2, pp. 229-250, 1995.

[26] H. Kitajima, Y. Katsuta, and H. Kawakami, "Bifurcations of periodic solutions in a coupled oscillator with voltage ports," IEICE Trans. Fundamentals, vol. 81, no. 3, pp. 476-482, 1998.

[27] T. Ueta and H. Kawakami, "Bifurcation in asymmetrically coupled BVP oscillators," Int. J. Bifurcation and Chaos, vol. 13, no. 5, pp. 1319-1327, 2003.

[28] Y. Horikawa and H. Kitajima, "Transient chaotic rotating waves in a ring of unidirectionally coupled symmetric Bonhoeffer-van der Pol oscillators near a codimension-two bifurcation point," Chaos, vol. 22, no. 3, 033115, 2012.

[29] H. Kawakami, Nonlinear Phenomena, http://cms.db.tokushima-u.ac.jp/DAV/lecture/125260/ LectureNote/2006/NonlinearPhenomena.pdf (in Japanese).

[30] H. Kawakami and T. Ueta, Chaos CG by Programming Language C, Saiensu-sha Co., Tokyo, 1994 (in Japanese).

[31] M. Rodriguez, F. Blesa, and R. Barrio, "OpenCL parallel integration of ordinary differential equations: Applications in computational dynamics," Computer Physics Communications, vol. 192, pp. 228-236, 2015. 